\title{
A Novel Flow-Diverting Device (Tubridge) for the Treatment of 28 Large or Giant Intracranial Aneurysms: A Single-Center Experience
}

\author{
Y. Zhou, P.-F. Yang, Y.-B. Fang, Y. Xu, B. Hong, W.-Y. Zhao, Q. Li, R. Zhao, Q.-H. Huang, and J.-M. Liu
}

\begin{abstract}
BACKGROUND AND PURPOSE: The Tubridge flow diverter is a novel device developed in China and aimed at reconstructing the parent artery and occluding the aneurysm. We conducted this study to evaluate its feasibility, safety, and efficacy for the treatment of large or giant internal carotid artery aneurysms, which are still challenging with conventional therapy.
\end{abstract}

MATERIALS AND METHODS: The clinical and angiographic data of 28 patients with 28 large or giant internal carotid artery aneurysms treated with Tubridge flow diverters were prospectively collected and analyzed.

RESULTS: Thirty-three Tubridge flow diverters were successfully implanted except for 1 poor midstent opening; the result was a technical success rate of $97.0 \%$ (32/33). Follow-up angiographies were available for 25 aneurysms; the mean follow-up was 9.9 months (5-24 months). Of the 25 aneurysms, 18 (72.0\%) were completely occluded, 6 (24.0\%) were improved, and 1 (4.0\%) was unchanged. All of the visible covered branches and parent arteries were patent, with no stenosis or obliteration. During a follow-up of 6-30 months (mean, 19 months), symptoms were resolved in 13 patients, improved in 6 patients, and unchanged in 4 patients. Five patients experienced transient clinical deterioration due to a postoperative increased mass effect. Procedure-related morbidity and mortality were both zero.

CONCLUSIONS: Our preliminary experience shows that the Tubridge flow diverter is a safe and effective tool for treating large and giant internal carotid artery aneurysms. However, multicenter randomized trials and studies involving a long-term follow-up are necessary.

ABBREVIATIONS: $\mathrm{FD}=$ flow diversion; $I \mathrm{D}=$ inse diameter

arge and giant aneurysms are associated with much worse outComes than small ones. ${ }^{1}$ The treatment of large and giant aneurysms is challenging for both neurosurgeons and neurointerventionalists, either by conventional endovascular treatment or surgery. Deconstructive approaches, such as ICA occlusion, require sufficient compensation from other blood vessels. Even when this criterion is satisfied, the rate of ischemia has been re-

Received November 12, 2013; accepted after revision February 3, 2014

From the Department of Neurosurgery, Changhai Hospital, Second Military Medical University, Shanghai, P.R. China.

Yu Zhou and Peng-Fei Yang contributed equally to this work.

This work was supported by the Subproject of National Technology Supporting Program (2011BAI08B14), Logistics Scientific Research Project (BWS11C008), and the Science and Technology Commission of Shanghai Municipality (10441901902, 10DZ0510800).

Please address correspondence to Qing-Hai Huang, MD, Department of Neurosurgery, Changhai Hospital, Second Military Medical University, 168 Changhai Rd, Shanghai 200433, China; e-mail: ocinhqh@163.com; and Jian-Min Liu, MD, Department of Neurosurgery, Changhai Hospital, Second Military Medical University, 168 Changhai Rd, Shanghai 200433, China; e-mail: Chstroke@163.com

--m Indicates open access to non-subscribers at www.ajnr.org

http://dx.doi.org/10.3174/ajnr.A3925 ported to be as high as $4 \%-15 \% .^{2}$ In addition, there is a chance of de novo aneurysm following ICA occlusion. ${ }^{3}$ Bypass surgery, especially high-flow bypass, preceding ICA occlusion may help to reduce the occurrence of ischemic complications, but operative complications are not uncommon, though the rate varies in different articles. ${ }^{4}$ In contrast, reconstructive approaches aimed at preserving the parent artery were associated with greatly increased recanalization rates that were reported to range from $19.2 \%$ to $50 \%{ }^{2,5}$

The Tubridge is a new type of flow-diversion (FD) device developed by MicroPort Medical Company (Shanghai, China) on the basis of our previous hemodynamic studies of intracranial aneurysms, ${ }^{6}$ aimed at treating complex aneurysms that were difficult to access via clipping or conventional endovascular treatment, such as large and giant aneurysms, and providing more treatment options for neurointerventionalists and neurosurgeons. After demonstrating its efficacy and safety in animal experiments, ${ }^{7,8}$ we obtained the consent of the Ethics Committee and China Food and Drug Administration to initiate the present prospective clinical trial, aimed at evaluating its feasibility, safety, and efficacy for the treatment of large or giant internal carotid artery aneurysms. 


\section{MATERIALS AND METHODS}

\section{Patient Selection and Population}

After obtaining approval from the Ethics Committee and China Food and Drug Administration, we conducted this prospective study. The inclusion criteria for Tubridge flow-diverter placement were as follows: 1) The subject understands the nature of the procedure and provides written informed consent; 2) the subject is willing to return to the investigational site for the 30-day and 6-month follow-up evaluations, at least; 3 ) the subject is $18-75$ years of age; 4) the subject has $\geq 1$ wide-neck aneurysm (neck diameter of $\geq 4.0 \mathrm{~mm}$ or dome/neck ratio of $\leq 1$ ); 5 ) saccular aneurysms are $\geq 10 \mathrm{~mm}$; 5 ) the diameter of the parent artery is 2.0-6.5 $\mathrm{mm}$; and 6) the internal carotid artery aneurysm is unruptured. Aneurysms that were ruptured, dissecting, or comorbid with other intracranial diseases were excluded. Recanalized large or giant aneurysms were also included in this study. Therapeutic alternatives, including conventional endovascular treatment or flow-diversion treatment, were considered by the authors of the present article to determine the most appropriate course of treatment. Written consent was obtained from each patient.

Overall, 28 patients with 28 large or giant aneurysms were recruited between August 2010 and August 2012 (2 patients had multiple aneurysms, which were located at the opposite side of the target aneurysms, and were not included in this study). The group comprised 8 men and 20 women; the mean age was 54.8 years (range, 20-73 years). The clinical presentations of these patients varied and included no symptoms $(n=6)$, headache $(n=11)$, recanalization $(n=3)$, oculomotor paralysis $(n=7)$, blurred vision $(n=3)$, blindness $(n=1)$, and amenorrhea $(n=1)$. Endovascular treatments were performed by 2 authors of the present article (J.-M.L. and Q.-H.H), each of whom has $>10$ years of experience in intracranial stent placement. Of the 3 recanalized aneurysms, 1 case was treated with coils alone, and the remaining 2 cases were treated with stent-assisted coiling with either Neuroform (Stryker Neurovascular, Fremont, California) or LEO (Balt Extrusion, Montmorency, France).

\section{Aneurysm Morphology}

Locations of the 28 aneurysms were defined on the basis of the segment of the ICA (according to the classification of Bouthillier et $\mathrm{al}^{9}$ ) where the aneurysm necks were found. Thus, 1 of the 28 aneurysms was located at the petrous segment (the ICA within the carotid canal), 10 were located in the cavernous segment (the ICA from the superior margin of the petrolingual ligament to the proximal dural ring), 7 were located in the paraclinoid segment (the ICA that begins at the proximal dural ring and ends at the distal dural ring where the ICA becomes intradural), 8 were located in the ophthalmic segment (the ICA that begins at the distal dural ring and ends just proximal to the origin of the posterior communicating artery), and 2 were located in the communicating segment (the ICA that begins just proximal to the origin of the posterior communicating artery and ends at the ICA bifurcation). The size of the aneurysms ranged from 11.3 to $44 \mathrm{~mm}$, and the mean size was $21.6 \mathrm{~mm}$. The aneurysms were further classified according to size as large $(10-15 \mathrm{~mm} ; n=4)$, very large $(15-25$ $\mathrm{mm} ; n=16$ ), or giant ( $\geq 25 \mathrm{~mm} ; n=8)$. The diameters of the proximal parent arteries ranged from 3.6 to $5.8 \mathrm{~mm}$. As described above, 3 aneurysms had been treated with coiling or stent-assisted coiling before, while the others were all treated for the first time.

\section{Description of the Tubridge Flow Diverter and the Procedure}

The Tubridge flow diverter is a braided, self-expanding stentlike device with flared ends. Current Tubridge flow diverters are available in various diameters $(2.5-6.5 \mathrm{~mm})$ and lengths $(12-45 \mathrm{~mm})$. The large Tubridge (diameter, $\geq 3.5 \mathrm{~mm}$ ) is a braid of 62 nickel-titanium microfilaments and 2 platinumiridium radio-opaque microfilaments; the small Tubridge (diameter, $<3.5 \mathrm{~mm}$ ) is composed of 46 nitinol and 2 platinumiridium microfilaments. All Tubridge flow diverters were designed with a pore size of $0.040-0.050 \mathrm{~mm}^{2}$ at the nominal diameter to provide high metal coverage (approximately $30.0 \%-$ $35.0 \%)$ at the aneurysmal neck after full opening.

The Tubridge is mounted to a delivery wire and constrained within a removable sheath. The tip of the delivery wire is J-shaped, which is designed to help prevent vascular endothelial cell injury and to facilitate microcatheter removal through previous devices and to deploy a second flow diverter. During the implantation procedure, a Tubridge-compatible standard 0.029 inse diameter (ID) microcatheter was placed in the distal segment (approximately $30 \mathrm{~mm}$ ) of the aneurysm neck with the assistance of a microwire. Then the Tubridge flow-diversion device was introduced via the microcatheter into the target zone. Manipulation during releasing resembles that in Silk flow diverters (Balt Extrusion). After we placed the Tubridge into position by pushing the delivery wire and simultaneously withdrawing the microcatheter, the device began to expand in the artery and was deployed. To increase the proportion of metal coverage at the aneurysm neck, we deliberately pushed the microcatheter toward the aneurysm neck while the aneurysm neck was partially covered by the stent. Generally, the shortening rate after complete deployment of the Tubridge is approximately $<50.0 \%$, depending on the size of the Tubridge relative to the vessel and any discrepancies between the proximal and distal vessel diameters. There is a marker in the middle of the Tubridge; the device can be retracted until released to that point. After one deploys the first one, a second flow diverter would be considered if a disturbed inflow jet was not observed.

Seven of the 25 initially treated aneurysms and all of the 3 recanalized aneurysms were treated by using the Tubridge alone; the remaining 18 aneurysms were treated with the Tubridge flow diverter and loose coiling. In the 18 patients treated with a combination of the Tubridge and coils, all flow diverters were deployed after coiling. In 1 aneurysm with an extremely wide neck, 1 LEO stent was deployed first, which was followed by the telescopic placement of 2 Tubridge flow diverters that were delivered through the LEO stent. After the procedure was completed, DynaCT (Siemens, Erlangen, Germany) reconstruction was performed in each patient to ensure the full opening of the Tubridge. To assess the friction associated with the delivery, after the procedure, the 2 operators were required to record their assessments of the delivery process compared with that in Enterprise stents (Codman \& Shurtleff, Raynham, Massachusetts), which were classified as "difficult," "comparable," and "better."

AJNR Am J Neuroradiol 35:2326-33 Dec 2014 www.ajnr.org 


\section{Anticoagulation and Antiplatelet Management}

Each patient received systemic heparin after the placement of the sheath. The activated clotting time was maintained at 2-3 times the baseline throughout the procedure. Each patient received dual antiplatelet drugs $(300 \mathrm{mg} /$ day aspirin plus $75 \mathrm{mg} /$ day clopidogrel) for at least 3 days before the procedure. A postoperative antiplatelet regimen was administered as follows: $<6$ weeks: 300 mg aspirin $+75 \mathrm{mg}$ clopidogrel; 6 weeks to 3 months: $100 \mathrm{mg}$ aspirin $+75 \mathrm{mg}$ clopidogrel; $\geq 3$ months: $100 \mathrm{mg}$ aspirin indefinitely.

\section{Clinical and Angiographic Evaluation}

According to our protocol, each patient was clinically evaluated at discharge and prescribed follow-up assessments at 1, 3, and 6 months posttreatment and yearly thereafter. An angiographic evaluation consisting of digital subtraction angiography was performed immediately after the procedure, at 6 months posttreatment, and yearly thereafter. For aneurysms treated with the Tubridge flow diverter plus coils, the angiographic results obtained immediately after the procedure were classified according to the Raymond classification system. For aneurysms treated with the Tubridge alone, flow modifications were defined as disrupted inflow jet, slow flow (if the contrast circulation within the aneurysm became slower), or reduced contrast filling (if increased contrast stagnation was observed within the aneurysm at the late venous phase of the angiographic series). ${ }^{10}$ At follow-up, the angiographic results were independently interpreted by 2 experienced neurosurgeons who were not involved in this study and compared with the initial results to determinate whether the aneurysms were completely occluded, improved, stable, or recanalized. Branches that were covered by flow diverters were examined to confirm patency. Each patient's clinical symptoms were evaluated and recorded as completely/partially relieved or worsening.

\section{RESULTS}

The characteristics of the 28 patients treated with Tubridge flow diverters, including demographic information, clinical presentation, morphologic features of the aneurysms, treatment results, and follow-up data are summarized in Tables 1 and 2 .

\section{Immediate Angiographic and Clinical Results}

Tubridge delivery was successful in all 28 patients. Thirty-three Tubridge flow diverters were implanted, all of which had a large (diameter, $\geq 3.5 \mathrm{~mm}$ ) braid of 64 microfilaments. For 5 aneurysms, 2 overlapping flow diverters were deployed, while the remaining 23 aneurysms were treated with a single flow diverter. No obvious difficulties with device delivery or deployment were encountered, and the amount of friction associated with the delivery was comparable with that of Enterprise stents. However, poor midstent opening was identified in 1 patient $(1 / 33,3.0 \%)$ with an aneurysm in the cavernous segment of the internal carotid artery and a remarkable tortuous parent artery. We attempted to expand the poorly opened stent with a microballoon; however, delivery of the microcatheter though the stent proved to be difficult, and the attempt was abandoned. Fortunately, this patient experienced no untoward effects of the procedure, and close clinical observation was arranged (Fig 1).
Table 1: Clinical, angiographic, and follow-up data in 28 patients with 28 large or giant ICA aneurysms

Characteristics

\begin{tabular}{lc}
\hline Patients (aneurysms) (No.) & $28(28)$ \\
Mean age (range) (yr) & $54.8(20-73)$ \\
Male/female & $8: 20$ \\
Aneurysm location & 2 \\
ICA communicating & 8 \\
ICA ophthalmic & 7 \\
ICA paraclinoid & 10 \\
ICA cavernous & 1 \\
ICA petrous & \\
Presentation & 6 \\
$\quad$ Asymptomatic & 11 \\
Headache & 3 \\
Recanalization & 7 \\
Oculomotor paralysis & 3 \\
Blurred vision & 1 \\
Blind & 16 \\
Amenorrhea & 8 \\
Aneurysm size & 1 \\
Large (10-15 mm) & \\
Very large (15-25 mm) & \\
Giant ( $\geq 25$ mm) & \\
\hline
\end{tabular}

Table 2: Clinical, angiographic, and follow-up data in 28 patients with 28 large or giant ICA aneurysms

\begin{tabular}{lc}
\hline \multicolumn{1}{c}{ Treatment } \\
\hline Treatment strategy \\
FD alone \\
FD + loose coiling & 10 \\
No. implanted flow diverters & 18 \\
$\quad$ Two overlapping flow diverters & \\
$\quad$ Single flow diverter & 5 \\
Technique adverse event & 23 \\
$\quad$ Poor midstent opening & 1 \\
Immediate angiographic results & \\
Partial occlusion & 16 \\
Neck remnant & 2 \\
Disrupted inflow jet and slow flow & 9 \\
Flow reduction & 7 \\
No change & 3 \\
Clinical symptoms & \\
Resolved & 13 \\
Improved & 6 \\
No change & 4 \\
Transient worsening & 5 \\
Overall procedure-related morbidity/mortality & $0 / 0$ \\
Follow-up angiographic results $(n=25)$ & $18(72 \%)$ \\
Complete occlusion & $6(24 \%)$ \\
Improvement & $1(4 \%)$ \\
Stable & \\
\hline
\end{tabular}

For the 18 aneurysms treated with a flow diverter and coiling, the immediate angiographic results included neck remnant in 2 aneurysms and sac residue in the remaining 16 aneurysms. For the 10 aneurysms treated with a flow diverter alone, disrupted inflow jet and slow flow were observed in each patient except for the 1 patient with a poor midstent opening; flow reduction was observed in 7 aneurysms (reduction of $<50.0 \%$ in 5 patients, reduction of $\geq 50.0 \%$ in 2 patients) (Fig 2), and the remaining 3 aneurysms exhibited no obvious change ( 1 was due to poor midstent opening as described above). In total, there were 27 visible branches covered by the Tubridge, including 23 ophthalmic ar- 

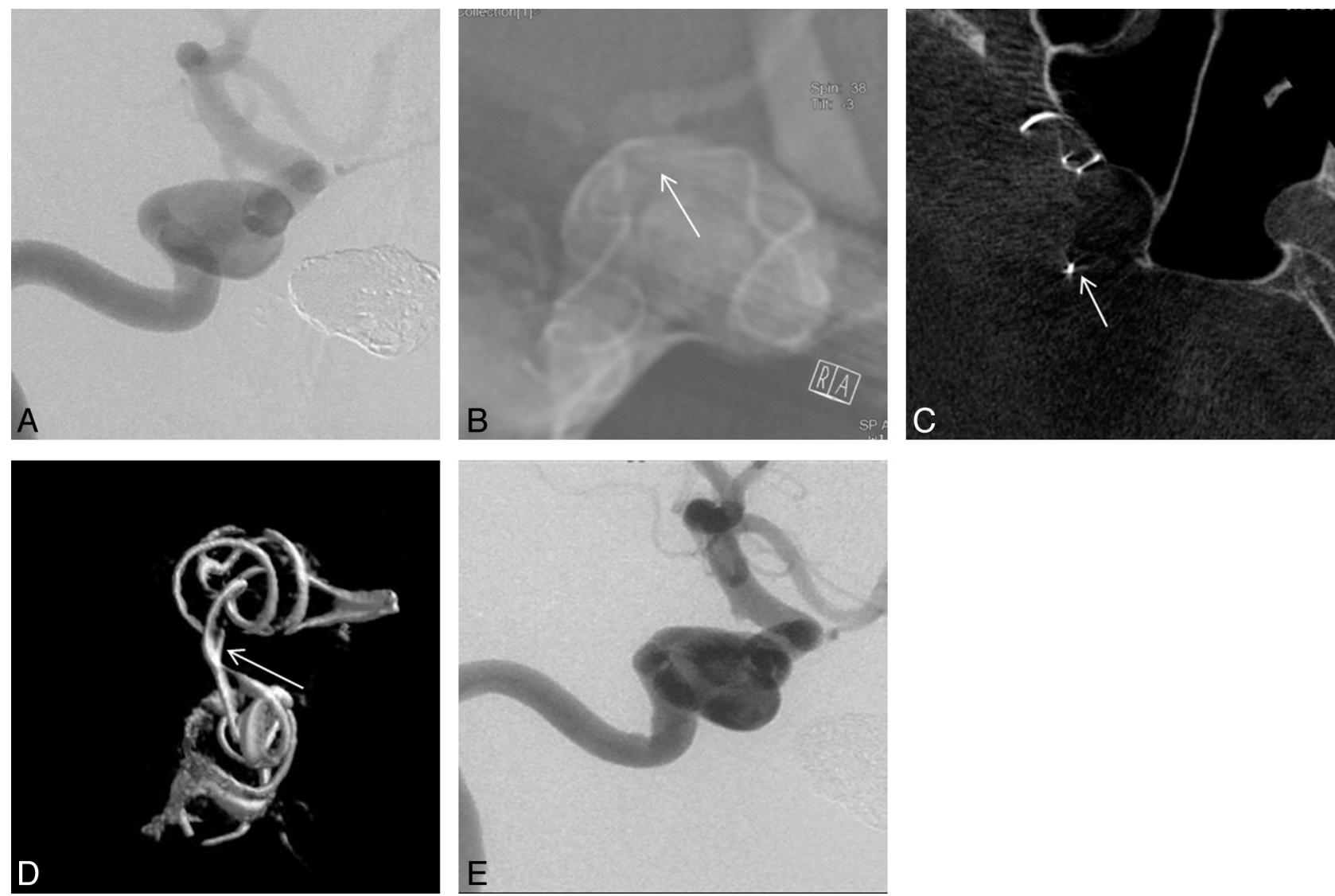

FIG 1. Right internal carotid artery digital subtraction angiography $(A)$ revealed a 13-mm wide-neck cavernous segment aneurysm. A Tubridge flow diverter was deployed into the parent artery, aimed at covering the aneurysmal neck, but postoperative DynaCT revealed that the flow diverter was not fully opened $(B$, white arrow). Cross-sectional image $(C$, white arrow) and 3D reconstruction $(D$, white arrow) show poor opening of the device. Fortunately, 18-month follow-up angiography reveals that the aneurysm is stable with a patent artery $(E)$.

teries, 2 meningohypophyseal trunks, 1 early frontal branch (in this case, the Al segment was absent, and the distal part of the Tubridge was deployed into the middle cerebral artery), and 1 posterior communicating artery, all of which were patent following Tubridge deployment.

\section{Clinical Outcome}

In all 28 patients, neither ischemic nor hemorrhagic complications occurred. During the follow-up period of 6-30 months (mean, 19.0 months), symptoms were resolved in 13 patients (4 cases of oculomotor paralysis, 9 headaches), improved in 6 patients ( 3 cases of oculomotor paralysis, 1 case of blurred vision, 2 headaches), and unchanged in 4 patients ( 2 cases of blurred vision, 1 case of blindness, and 1 case of amenorrhea). Five patients experienced transient clinical deterioration due to a postoperative increase in mass effect. Of these 5, three had moderate headache within 2 weeks after the operation, and 2 experienced worsening oculomotor nerve paralysis within 3 months of the procedure. However, all of these symptoms had resolved by the last follow-up. Thus, there were no instances of procedure-related morbidity or mortality.

\section{Follow-Up Angiographic Results}

Twenty-five patients with 25 aneurysms completed a least 1 follow-up cerebral angiography; the remaining patients were unwilling to undergo angiographic follow-up for various reasons. The follow-up periods ranged from 5 to 24 months (mean, 9.9 months). Eighteen of the 25 (72.0\%) aneurysms for which follow-up data were available were completely occluded, whereas the $6(24.0 \%)$ in which only a neck remnant was observed (Fig 3 ) were improved, and 1 (the case in which the stent did not fully open) remained unchanged. Detailed outcomes of aneurysms treated with "FD alone" and "FD plus coiling" are shown in Table 3. In the "FD alone" group, the complete occlusion rate was $75.0 \%$, while that in the "FD plus coiling" group was $70.5 \%$. On the angiograms, parent arteries of all cases were patent, and there was no evidence of intimal hyperplasia or in-stent stenosis. All of the visible covered branches were patent without stenosis or obliteration.

\section{DISCUSSION}

In the present article, we report preliminary findings related to the application of Tubridge flow diverters in large or giant ICA aneurysms. Despite the complex morphologies of these aneurysms, the short-term results are satisfactory, with a high technique success rate and minor technique/clinical complications.

\section{Characteristics and Feasibility of Tubridge Flow Diverters}

The Tubridge is actually a stentlike vessel-reconstruction device designed with a high metal coverage rate and low porosity. It diverts blood flow away from the aneurysm while preserving normal blood flow of the side branches.

AJNR Am J Neuroradiol 35:2326-33 Dec 2014 www.ajnr.org 

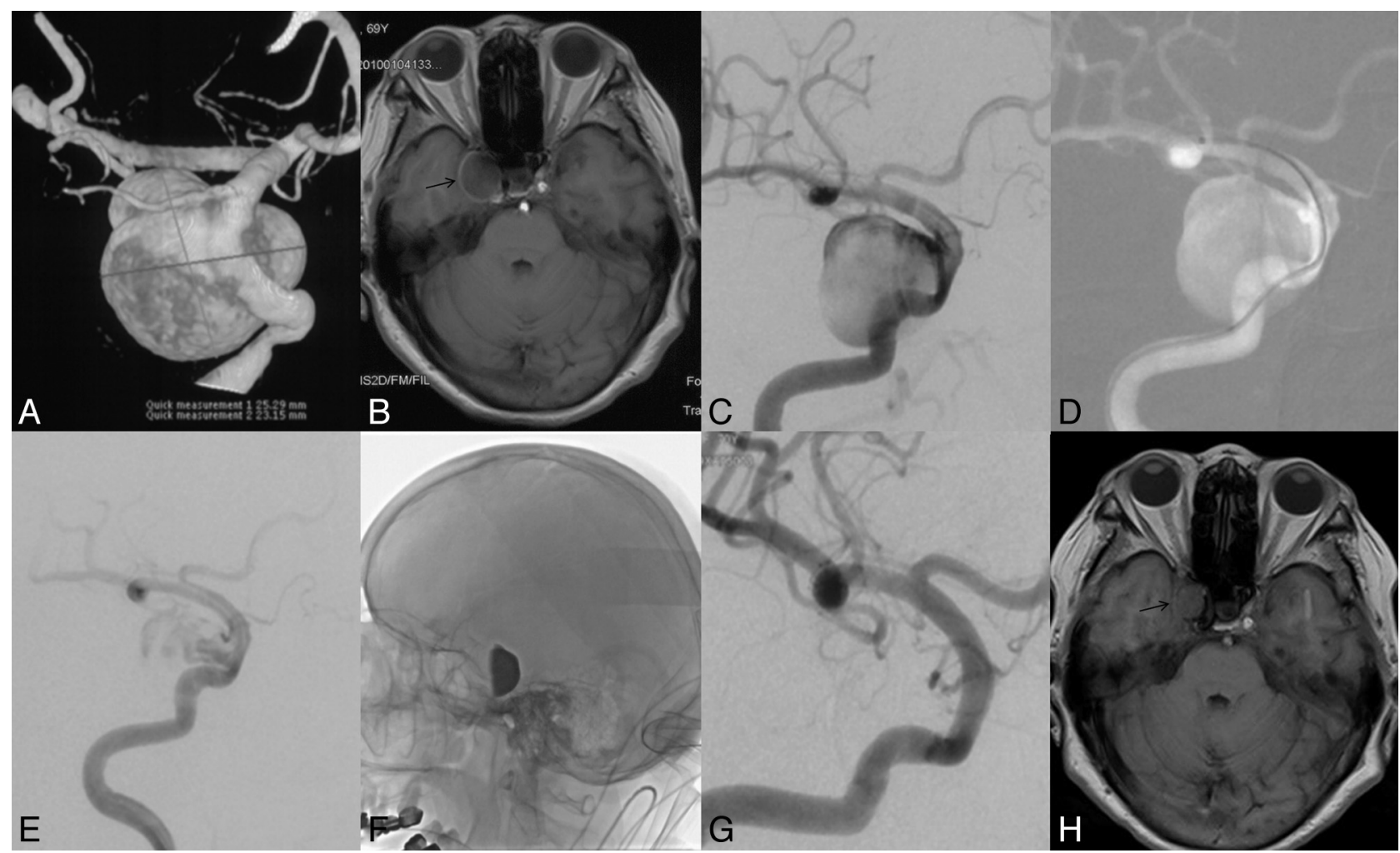

FIG 2. Right internal carotid artery digital subtraction angiography and $3 D$ reconstruction $(A-C)$ reveal a giant cavernous segment aneurysm of approximately $25.3 \mathrm{~mm}$. The microcatheter is delivered across the aneurysmal neck $(D)$, and 1 Tubridge flow diverter is deployed. Postoperative angiography reveals a disrupted inflow jet, slow flow, and flow reduction $(E$ and $F$ ). The 23-month follow-up angiography reveals that the aneurysm is completely occluded $(G)$. $H$, The black arrow points to the patent covered ophthalmic artery during follow-up compared with the preoperative image. MR imaging follow-up shows shrinkage of the aneurysm (black arrow).
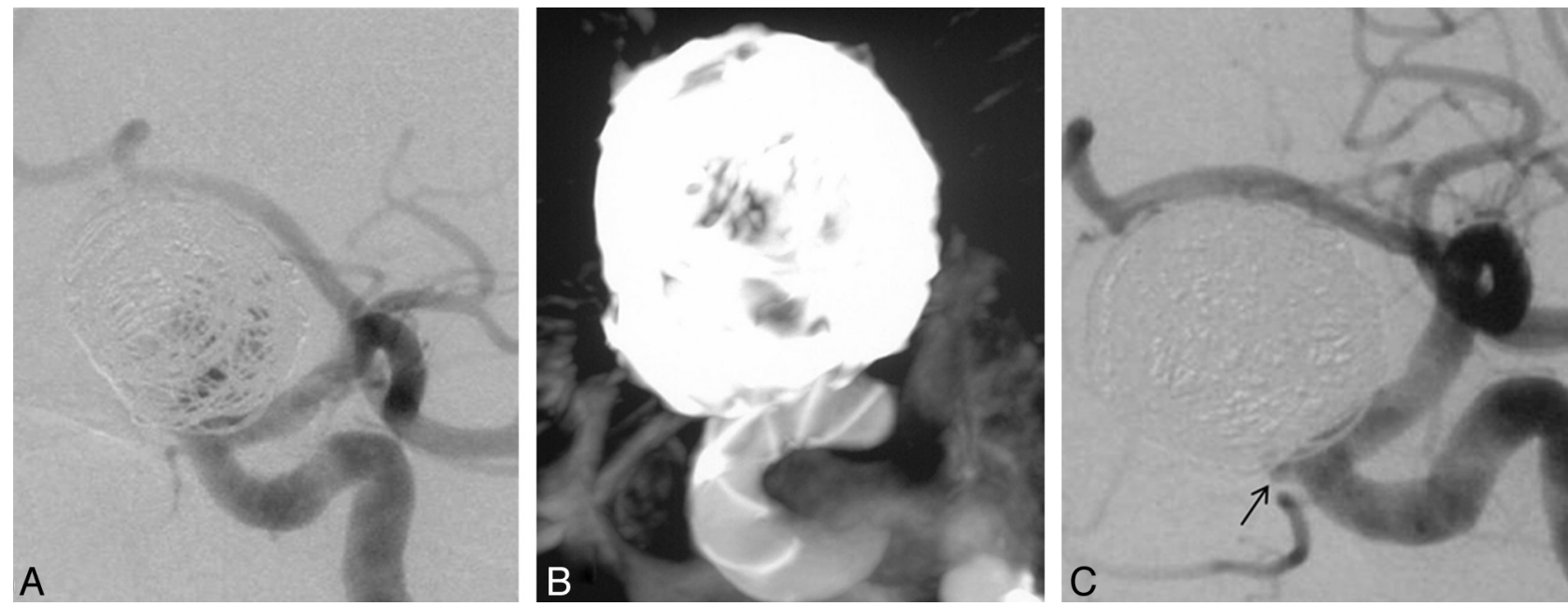

FIG 3. Left internal carotid artery digital subtraction angiography of an aneurysm treated with a Tubridge flow diverter combined with coils, which results in partial occlusion of the aneurysm $(A)$. DynaCT reconstruction reveals full opening of the flow diverter $(B)$.The 18 -month follow-up angiography reveals that the aneurysm has improved, and only a neck remnant is observed (C, black arrow).

Table 3: Follow-up angiographic outcome of different treatment modalities

\begin{tabular}{lccc}
\hline & \multicolumn{3}{c}{ FD Plus } \\
& FD Alone & Coiling & Total \\
\hline Complete occlusion & $6(75.0 \%)$ & $12(70.5 \%)$ & $18(72.0 \%)$ \\
Improved to neck remnant & $1(12.5 \%)$ & $5(29.4 \%)$ & $6(24.0 \%)$ \\
Unchanged & $1(12.5 \%)$ & 0 & $1(2.0 \%)$ \\
Total & 8 & 17 & 25 \\
\hline
\end{tabular}

Except for the use of a nickel-titanium alloy (commonly known as nitinol, which exhibits shape-memory and superelasticity) and flared ends, the Tubridge offers multiple structural improvements over the Pipeline Embolization Device (Covidien, Irvine, California) and Silk flow diverters (Table 4). The platinum-iridium material used for the radio-opaque microfilaments improves the visualization of both the diameter and the length during the placement procedure. More important, the use of 
Table 4: Structure comparison among different flow diverters

\begin{tabular}{|c|c|c|c|c|c|c|c|}
\hline \multirow[b]{2}{*}{ Type } & \multirow[b]{2}{*}{ Size (mm) } & \multicolumn{2}{|c|}{ Braided Microfilaments $^{a}$} & \multirow{2}{*}{$\begin{array}{l}\text { Radio-Opaque } \\
\text { Microfilaments }\end{array}$} & \multirow[b]{2}{*}{ Flared End } & \multirow{2}{*}{$\begin{array}{l}\text { Metal } \\
\text { Coverage }\end{array}$} & \multirow[b]{2}{*}{ Retrievable } \\
\hline & & No. & Material & & & & \\
\hline Pipeline & $3-5.5$ & 48 & $\begin{array}{l}75 \% \text { Cobalt chromium } \\
\text { and } 25 \% \text { platinum }\end{array}$ & NA & No & $30 \%-35 \%$ & $\mathrm{No}^{\mathrm{b}}$ \\
\hline Silk & $2.5-5$ & 48 & Nickel-titanium alloy & 4 Platinum wires & Yes & $35 \%-55 \%$ & Yes \\
\hline Surpass & $2.5-5$ & $\begin{array}{l}2.5 \mathrm{~mm}, 36 ; 3 \text { and } \\
\quad 4 \mathrm{~mm}, 60 ; 5 \mathrm{~mm}, 84\end{array}$ & Cobalt-chromium & 12 Platinum wires & No & $30 \%$ & NA \\
\hline Tubridge & $2.5-6.5$ & $\begin{array}{l}<3.5 \mathrm{~mm}, 46 \\
\quad \geq 3.5 \mathrm{~mm}, 62\end{array}$ & Nickel-titanium alloy & $\begin{array}{l}2 \text { Platinum-iridium } \\
\text { wires }\end{array}$ & Yes & $30 \%-35 \%$ & Yes \\
\hline
\end{tabular}

Note:-NA indicates not applicable.

${ }^{a}$ Braided microfilaments mean those main wires excluding microfilaments especially for radio-opaque usage.

${ }^{\mathrm{b}}$ The Pipeline Embolization Device is not retrievable, but at any point up to final deployment, it may be captured and removed from the body.

more braided microfilaments for the large-size flow diverter decreases the shortening rate of the flow diverter after full opening and offers more appropriate pore attenuation compared with the 48-wire design of the Silk or Pipeline flow diverters. Similarly, the recently introduced Surpass flow diverter (Stryker Neurovascular) was also designed with different structures: The 2.5-mm-diameter device has 48 wires, whereas the 3 - and 4-mm devices have 72 wires and the 5 -mm device is constructed of 96 wires. ${ }^{11}$ Moreover, their users experienced neither the complications of device migration nor incomplete neck coverage due to device shortening as reported in early series. ${ }^{10,12}$ We believe that these structural improvements combined with extensive operator expertise (both of our operators had practiced the delivery and deployment of the Tubridge on models and gained full knowledge of the Tubridge characteristics before placing it in humans) reduced the chance of technical adverse events in patients treated with FD.

A major concern during Tubridge development was related to theoretic difficulties of placing a device with more braided microfilaments. However, no remarkable delivery difficulties occurred. In the present trial, all flow diverters were successfully deployed except for 1 ; this outcome resulted in a technical adverse events rate of 3.0\%. Additionally, no flow diverters fell into the aneurysmal sac or missed complete neck coverage due to device shortening. These results are comparable with those reported in other FD series; technical adverse events rates for the Pipeline and Silk flow diverters have been reported as $2.3 \%-13 \%$ and $12.3 \%-23.1 \%$, respectively. ${ }^{10,12-23}$

In theory, a flow diverter can promote healing in aneurysms without additional coiling. However, coils were introduced into the aneurismal sac simultaneously with flow diverter placement in 18 aneurysms of this series. This was done to help the microcatheter cross the neck of extremely wide-neck aneurysms and/or to promote aneurysm thrombosis to prevent early or delayed aneurysm rupture. ${ }^{20}$ Additionally, for extremely wide-neck aneurysms, coils may provide good support for implanted flow diverters and help stabilize a flow diverter during deployment. Otherwise, flow diverters may protrude into the aneurismal sac due to the impact of blood flow.

\section{Safety of Tubridge Flow Diverters}

Although various articles have reported promising results from treating aneurysms with $\mathrm{FD}$, increasing questions have arisen about this type of treatment. In some prospective studies $^{13,16,18,19,21-26}$ and series with large numbers ${ }^{12}$ of the Pipeline and Silk devices, the periprocedural complication rate has been reported to generally range from $2.8 \%$ to $11 \%$, with a rate of ischemia and SAH/intracerebral hemorrhage ranging from $0.9 \%$ to $7.7 \%$ and $0 \%$ to $6.6 \%$, respectively. Delayed complication rates of ischemia, bleeding, and mass effect were reported to be $0 \%-$ $11.5 \%, 0 \%-4.7 \%$, and $0 \%-23 \%$, respectively. The overall morbidity and mortality associated with Pipeline and Silk in these studies were $0 \%-15 \%$ and $0 \%-6.6 \%$, respectively. Ischemic complications are generally due to intrastent thrombosis and/or side branch occlusion. However, intrastent thrombus formation and subsequent parent artery occlusion were always related to poor stent opening. ${ }^{12-17,26}$ Resistance to antiplatelets is another possible reason; however, for patients with full opening of the stents, the ischemic event rate was not reported to be higher than that for the self-expansion stent, ${ }^{12-17,26}$ though the dose of dual antiplatelet medicine varied in different articles.

To date, most side branch occlusion events have been reported to occur in patients who are treated with more than 2 flow diverters or stents: Szikora et $\mathrm{al}^{22}$ reported 2 cases of ophthalmic artery occlusion, in which the number of flow diverters implanted was either 3 or 4 . One patient in the Pipeline Embolization Device for the Intracranial Treatment of Aneurysms Trial experienced lenticulostriate occlusion after the implantation of 2 Pipeline flow diverters and 1 Neuroform stent. ${ }^{21}$ In our series, only 1 instance of poor stent opening occurred, which did not lead to occlusion of the parent artery. Moreover, we did not encounter any other periprocedural or delayed ischemic events by using the present antiplatelet regimen. To ensure subject safety in our series, we implanted no more than 2 flow diverters into any single parent artery. In addition, manipulation of the microcatheter and FD can help decrease the metal coverage rate on the aneurysmal neck and minimize the impact of diversion on the intact vessels and perforators. No visible side-branch occlusions were noted in the present series.

Another severe complication associated with flow diverter implantation is intracranial bleeding. Although the exact reason is unknown, some authors believe that increased intrasaccular pressure and unstable thrombus formation may be involved. ${ }^{20,27} \mathrm{On}$ the basis of reports of these events, the Balt Extrusion Company released an urgent safety notice in 2010 , which advised that coils be used in combination with Silk implantation. However, in the present series, we did not encounter any hemorrhagic complications. This outcome may be due to the implantation of additional coils for selected aneurysms. Additional coils may promote thrombi formation in the aneurysm sac and decrease the risk of aneurysm rupture. However, the low rates of hemorrhagic events 
observed in the present study may be due to biases associated with small sample sizes. The necessity of additional coil embolization is under review by various randomized clinical trials such as the Efficacy Trial of Intracranial Aneurysm Treatment Using Two Different Endovascular Techniques. ${ }^{28}$ A multicenter prospective trail comparing Tubridge flow-diverting treatments with Enterprise stent-assisted coiling, which is also being conducted in China (registered on the Chinese Clinical Trial Registry: ChiCTRTRC-13003127), may provide more information about the safety of Tubridge implantation.

Large aneurysms, especially those that are very large or giant, are more often associated with thrombus-induced mass effect or perianeurysm edema following flow diverter implantation. However, most of these complications are transient. Byrne et $\mathrm{al}^{24}$ reported 4 cases of worsening cranial nerve palsy or brain stem compression symptoms due to delayed aneurysm thrombosis and mass effect in 4 patients. Of these 4 patients, 3 recovered and 1 with a basilar artery aneurysm died. Berge et al ${ }^{19}$ reported that 15 patients presented with headaches that were associated with worsening symptoms; however, all of these symptoms were transient and eventually resolved. We also observed this phenomenon in 5 of our patients. However, all symptoms had resolved by the follow-up. Furthermore, we observed partial or complete improvement of the symptoms caused by mass effect in most of our patients, except for cases associated with badly damaged nerves.

\section{Efficacy of Tubridge Flow Diverter Implantation}

The concept of flow diversion relies on isolating blood flow away from the aneurysm sac and promoting reconstruction of the parent artery, rather than embolizing the aneurysm sac to make it as compact as possible. The immediate angiographic results often indicate a disturbed flow jet, contrast stagnation, or decreased contrast filling, but not complete occlusion (even in aneurysms treated with FD and coils). However, thrombosis will persist during the follow-up period. The available data suggest that the overall complete occlusion rates beyond 6 months for Pipeline and Silk are $68 \%-87.8 \%$ and $68.4 \%-94.4 \%$, respectively. ${ }^{12,16,19,25}$

In our series, we observed excellent angiographic results after Tubridge implantation. During the mean follow-up period of 9.9 months, 18 aneurysms were completely occluded. The overall complete occlusion rate was $72 \%$, which is comparable with those of silk flow diverters and the Pipeline Embolization Device and appears to be much better than those rates associated with conventional endovascular treatments. ${ }^{5,29,30}$ However, studies with a long-term follow-up and larger series are necessary. De Vries et $\mathrm{al}^{11}$ recently reported their 6-month follow-up result, which showed a complete occlusion (94\%), including 1 case with a 95\%$100 \%$ occlusion. However, their research contained a high number of small aneurysms, which may influence the occlusion rate. There are still 6 improved aneurysms in our series with only a neck remnant observed. These will be followed up to determine whether they will finally be completely occlude.

In this study, we listed the outcome of aneurysms treated with different modalities (Table 3). The FD-alone group seems to be comparable with the FD-plus coiling group; these results may raise doubts about the necessity of additional coils if there is no safety or delivery consideration as discussed above. However, the sample is small, and further study is needed to draw such conclusions.

\section{Limitations}

We acknowledge that the major limitations of this study include the relatively small series size, a short angiographic follow-up period, and lack of randomized comparisons with other potentially efficacious therapies. Patient-selection bias may also exist due to the strict inclusion criteria. However, the data suggest that the Tubridge flow diverter is a safe and effective tool for the treatment of large and giant ICA aneurysms.

Another concern is the application of an antiplatelet regimen for flow diverters. An appropriate regimen should minimize the risk of thromboembolic events, while using as low a dose of antiplatelet drugs as possible to avoid of hemorrhagic complications. However, antiplatelet regimens still vary in the literatures; the dose, the intervals, and even the main drug used are different. ${ }^{11,13,18,19,23,24}$ Our regimen is modified according to our previous experience of intracranial stent placement, which still seems to be safe for Tubridge implantation. However, multicenter randomized trials with larger subject numbers and long-term follow-up studies are necessary.

\section{CONCLUSIONS}

Our preliminary experience demonstrated that the Tubridge flow diverter is a safe and effective tool for the treatment of large and giant ICA aneurysms. However, multicenter randomized trials and long-term follow-up studies are necessary.

Disclosures: J.-M. Liu—RELATED: During the development, I gave some advice about the design of this device and offered some data about our previous hemodynamic studies.

\section{REFERENCES}

1. Barrow DL, Alleyne C. Natural history of giant intracranial aneurysms and indications for intervention. Clin Neurosurg 1995;42:214-44

2. Gonzalez NR, Duckwiler G, Jahan R, et al. Challenges in the endovascular treatment of giant intracranial aneurysms. Neurosurgery 2006;59:S113-24

3. Zhou Y, Yang P, Zhang Y, et al. Posterior cerebral artery-posterior communicating artery (PCA-PComA) aneurysms: report of five cases and literature review. Neurol India 2012;60:228-30

4. van Doormaal TP, van der Zwan A, Verweij BH, et al. Treatment of giant and large internal carotid artery aneurysms with a high-flow replacement bypass using the excimer laser-assisted nonocclusive anastomosis technique. Neurosurgery 2008;62 (6 suppl 3):1411-18

5. Gao X, Liang G, Li Z, et al. A single-centre experience and follow-up of patients with endovascular coiling of large and giant intracranial aneurysms with parent artery preservation. J Clin Neurosci 2012; 19:364-69

6. Huang QH, Yang PF, Zhang X, et al. Effects of flow diverter with low porosity on cerebral aneurysms: a numerical stimulative study [in Chinese]. Zhonghua Yi Xue Za Zhi 2010;90:1024-27

7. Hong $B$, Wang $K$, Huang Q, et al. Effects of metal coverage rate of flow diversion device on neointimal growth at side branch ostium and stented artery: an animal experiment in rabbit abdominal aorta. Neuroradiology 2012;54:849-55

8. Wang K, Huang Q, Hong B, et al. Correlation of aneurysm occlusion with actual metal coverage at neck after implantation of flow-diverting stent in rabbit models. Neuroradiology 2012;54:607-13 
9. Bouthillier A, van Loveren HR, Keller JT. Segments of the internal carotid artery: a new classification. Neurosurgery 1996;38:425-32

10. Lubicz B, Collignon L, Raphaeli G, et al. Pipeline flow-diverter stent for endovascular treatment of intracranial aneurysms: preliminary experience in 20 patients with 27 aneurysms. World Neurosurg 2011;76:114-19

11. De Vries J, Boogaarts J, Van Norden A, et al. New generation of flow diverter (Surpass) for unruptured intracranial aneurysms: a prospective single-center study in 37 patients. Stroke 2013;44:1567-77

12. O'Kelly CJ, Spears J, Chow M, et al. Canadian experience with the Pipeline embolization device for repair of unruptured intracranial aneurysms. AJNR Am J Neuroradiol 2013;34:381-87

13. Becske T, Kallmes DF, Saatci I, et al. Pipeline for uncoilable or failed aneurysms: results from a multicenter clinical trial. Radiology 2013;267:858-68

14. Yeung TW, Lai V, Lau HY, et al. Long-term outcome of endovascular reconstruction with the Pipeline embolization device in the management of unruptured dissecting aneurysms of the intracranial vertebral artery. J Neurosurg 2012;116:882-87

15. Wagner A, Cortsen M, Hauerberg J, et al. Treatment of intracranial aneurysms: reconstruction of the parent artery with flow-diverting (Silk) stent. Neuroradiology 2012;54:709-18

16. Velioglu M, Kizilkilic O, Selcuk H, et al. Early and midterm results of complex cerebral aneurysms treated with Silk stent. Neuroradiology 2012;54:1355-65

17. Tähtinen OI, Manninen HI, Vanninen RL, et al. The Silk flow-diverting stent in the endovascular treatment of complex intracranial aneurysms: technical aspects and midterm results in 24 consecutive patients. Neurosurgery 2012;70:617-23

18. Fischer S, Vajda Z, Aguilar Perez M, et al. Pipeline embolization device (PED) for neurovascular reconstruction: initial experience in the treatment of 101 intracranial aneurysms and dissections. Neuroradiology 2012;54:369-82

19. Berge J, Biondi A, Machi P, et al. Flow-diverter Silk stent for the treatment of intracranial aneurysms: 1-year follow-up in a multicenter study. AJNR Am J Neuroradiol 2012;33:1150-55

20. Turowski B, Macht S, Kulcsar Z, et al. Early fatal hemorrhage after endovascular cerebral aneurysm treatment with a flow diverter
(SILK-stent): do we need to rethink our concepts? Neuroradiology 2011;53:37-41

21. Nelson PK, Lylyk P, Szikora I, et al. The Pipeline embolization device for the intracranial treatment of aneurysms trial. AJNR Am J Neuroradiol 2011;32:34-40

22. Szikora I, Berentei Z, Kulcsar Z, et al. Treatment of intracranial aneurysms by functional reconstruction of the parent artery: the Budapest experience with the Pipeline embolization device. AJNR Am J Neuroradiol 2010;31:1139-47

23. Lubicz B, Collignon L, Raphaeli G, et al. Flow-diverter stent for the endovascular treatment of intracranial aneurysms: a prospective study in 29 patients with 34 aneurysms. Stroke 2010;41:2247-53

24. Byrne JV, Beltechi R, Yarnold JA, et al. Early experience in the treatment of intra-cranial aneurysms by endovascular flow diversion: a multicentre prospective study. PLoS One 2010;5:e12492

25. Lylyk P, Miranda C, Ceratto R, et al. Curative endovascular reconstruction of cerebral aneurysms with the Pipeline embolization device: the Buenos Aires experience. Neurosurgery 2009; 64:632-42

26. McAuliffe $\mathrm{W}$, Wycoco $\mathrm{V}$, Rice $\mathrm{H}$, et al. Immediate and midterm results following treatment of unruptured intracranial aneurysms with the Pipeline embolization device. AJNR Am J Neuroradiol 2012;33:164-70

27. Hassan T, Ahmed YM, Hassan AA. The adverse effects of flow-diverter stent-like devices on the flow pattern of saccular intracranial aneurysm models: computational fluid dynamics study. Acta Neurochir (Wien) 2011;153:1633-40

28. ClinicalTrials.gov. Multicenter Randomized Trial on Selective Endovascular Aneurysm Occlusion With Coils Versus Parent Vessel Reconstruction Using the SILK Flow Diverter (MARCO POLO PostMarket Clinical Investigation). http://www.clinicaltrials.gov/ct2/ show $/$ NCT01084681?term $=$ Marco + Polo\&rank $=1$. Accessed March 17,2014

29. Ferns SP, Sprengers ME, van Rooij WJ, et al. Coiling of intracranial aneurysms: a systematic review on initial occlusion and reopening and retreatment rates. Stroke 2009;40:e523-29

30. Sluzewski M, Menovsky T, van Rooij WJ, et al. Coiling of very large or giant cerebral aneurysms: long-term clinical and serial angiographic results. AJNR Am J Neuroradiol 2003;24:257-62 